\title{
Dynamic changes in the levels of maternal serum squamous cell carcinoma antigen, a potential biomarker of amniotic fluid embolism, before and after delivery in relation to the mode of delivery
}

\author{
KAZUTOSHI NAKANO ${ }^{1}$, NAOKI KAWAHARA ${ }^{1}$, YUKI YAMADA ${ }^{1}$, LIU LOTA $^{2}$, \\ CHIHO OHBAYASHI $^{2}$ and HIROSHI KOBAYASHI ${ }^{1,3}$
}

\begin{abstract}
Departments of ${ }^{1}$ Obstetrics and Gynecology, and ${ }^{2}$ Diagnostic Pathology, Nara Medical University, Kashihara, Nara 634-8522;
${ }^{3}$ Department of Obstetrics and Gynecology, Ms.Clinic MayOne, Kashihara, Nara 634-0813, Japan
\end{abstract}

Received September 22, 2020; Accepted January 21, 2021

DOI: $10.3892 /$ wasj.2021.89

\begin{abstract}
Amniotic fluid embolism (AFE) is a serious disease in which amniotic fluid components enter the maternal systemic circulation, causing cardiopulmonary collapse in pregnant women. It has been previously reported that amniotic fluid contains extremely high levels of squamous cell carcinoma antigen (SCCA), and that pregnant women who do not survive due to AFE have high blood SCCA levels. The aim of the present study was to determine the possible mechanisms through which SCCA in amniotic fluid enters the maternal blood, as well as the potential origin of SCCA. The prospective study included a cohort of 464 women (339 normal vaginal deliveries, 97 cesarean deliveries without labor, and 28 cesarean deliveries with labor). The dynamic changes in maternal serum SCCA levels were determined before and after delivery in relation to the mode of delivery, and SCCA levels were measured in the placenta, fetal skin, amniotic fluid cell components, amniotic fluid and neonatal urine. Serial serum samples collected at the time of admission, at $2 \mathrm{~h}$ postpartum, and on postpartum day 3 were quantitatively measured for SCCA by enzyme-linked immunosorbent assay. Amniotic fluid and neonatal urine SCCA levels were also measured. The protein expression of SCCA in the placenta and fetal skin was assessed by immunohistochemistry. In vaginal deliveries, there was a significant increase in serum SCCA levels from admission to $2 \mathrm{~h}$ postpartum, and SCCA levels decreased on postpartum day 3 . In cesarean deliveries, the SCCA levels at the time of admission and $2 \mathrm{~h}$ postpartum did not differ. The SCCA levels were significantly higher in
\end{abstract}

Correspondence to: Dr Hiroshi Kobayashi, Department of Obstetrics and Gynecology, Nara Medical University, 840 Shijo-cho, Kashihara, Nara 634-8522, Japan

E-mail: hirokoba@naramed-u.ac.jp

Key words: squamous cell carcinoma antigen, vaginal delivery, cesarean section, amniotic fluid, fetal urine women who underwent vaginal deliveries compared to those that underwent cesarean deliveries without labor $(\mathrm{P}=0.033)$. Immunohistochemical staining revealed no SCCA expression in the placenta and fetal skin. The SCCA levels in neonatal urine immediately after birth were as high as those in the amniotic fluid, suggesting that SCCA may originate from fetal urine. The present study thus suggests that amniotic fluid SCCA levels, which may be derived from fetal urine, can enter the maternal circulation during vaginal delivery. The onset of labor and full cervical dilatation are the main causes of entry of amniotic fluid components into the maternal circulation.

\section{Introduction}

Amniotic fluid embolism (AFE), a rare and severe obstetric complication, is a significant cause of maternal mortality worldwide (1). AFE occurs in a fetal antigen dose-dependent (mechanical obstruction subtype) or -independent manner (anaphylactic and anaphylactoid reaction subtype) (2). The former subtype is believed to occur when amniotic fluid disastrously enters the maternal circulation, resulting in the physical obstruction of pulmonary circulation. The latter subtype is predominantly mediated by the first exposure to amniotic fluid components that may cause an anaphylactoid reaction (3). Although AFE remains unpredictable based on current diagnostic strategies, recent advances in molecular biology have facilitated analysis of its pathogenesis. Advances in biochemistry experiments have enabled the identification of novel serum biomarkers of amniotic fluid passage into the maternal circulation (4-13). For example, Zinc-coproporphilin-1 (ZnCP1) (4), sialyl Tn (STN) $(5,6)$ and insulin-like growth factor binding protein 1 (IGFBP1) (12) have been reported as substances that are extremely abundant in amniotic fluid and present in trace amounts in maternal blood. In 2012, the authors newly identified squamous cell carcinoma antigen (SCCA) as a substance with a very high amniotic fluid/maternal blood concentration ratio (10). SCCA is a member of the serine protease inhibitor family of proteins, serpin, physiologically found in the spinous and granular layers of normal squamous epithelium $(3,14)$. 
SCCA is typically expressed in neoplastic cells of epithelial or endodermal origins, such as advanced squamous cell carcinomas of the cervix (14). SCCA inhibits cell damage caused by lysosomal cathepsins; however, the regulation of SCCA gene expression, its biological function and localization in each organ remain unclear (15). SCCA is present in trace amounts in peripheral blood even in non-pregnant women (14). Therefore, SCCA is not an amniotic fluid specific substance, but is characterized by the highest amniotic fluid/maternal blood concentration ratio, indicating that SCCA is suitable for serodiagnosis of AFE (10). This fact suggests that SCCA is a more valuable and reliable marker for serodiagnosis of AFE than ZnCP1, STN and IGFBP1 (9-11). In 2017, the authors reported extremely high levels of SCCA in the maternal serum of 4 patients with autopsy-proven AFE (13).

It was previously demonstrated that amniotic fluid enters the maternal circulation, particularly in the uterine vasculature, in a variety of obstetric disorders (16). Maternal intravascular fetal material during peripartum hysterectomy for the treatment of uterine rupture, abruption, uterine atony, placenta previa, placenta accreta, coagulopathy and retained placenta has been found to be present in one-third of patients (16). Furthermore, the fetal-to-maternal transfer of cell-free fetal DNA and fetal blood through the placenta and uterine vasculature may be common at some point during pregnancy (17). Therefore, the entry of amniotic fluid and fetal material into the maternal circulation and uterine vasculature is necessary (minimum requirement) for the onset of AFE, although it is not sufficient (2). Anaphylactic and anaphylactoid reactions can occur at the first exposure to amniotic fluid components (2).

The aim of the present study was to determine the dynamic changes in serum SCCA levels before and after delivery in relation to the mode of delivery and the potential origin of amniotic fluid SCCA. Maternal serum SCCA levels were measured before and after delivery to determine whether amniotic fluid components enter the maternal circulation only in patients with AFE or even in women with normal labor. Knowing whether amniotic fluid gains access to the systemic circulation during normal vaginal delivery is important for understanding the pathophysiology of AFE.

\section{Patients and methods}

Patient information. The collection of blood, amniotic fluid, urine and tissue samples was approved by the Institutional Review Board for Human Research at Nara Medical University (no. 579, 579-2, 873 and 873-2). Informed consent was obtained from all participants. The present prospective first cohort study was conducted at the Nara Medical University Hospital, Kashihara, Japan, from December, 2011 to October, 2015 to measure the dynamic changes in maternal serum SCCA levels before and after delivery in relation to the mode of delivery. Japanese pregnant women who had been admitted to the labor ward or operation room were recruited. All women had singleton pregnancies. Women with all levels of parity were included. Women undergoing instrumental delivery and those with pregnancy-related complications, including hypertensive disorders of pregnancy or preeclampsia were excluded. No participant had any malignant disease. Women who underwent normal vaginal delivery $(\mathrm{n}=339)$, planned cesarean section without labor $(n=97)$, or emergency cesarean section after the onset of labor $(\mathrm{n}=28)$ were included. The second cohort study was conducted to determine the potential origin of SCCA in amniotic fluid from September, 2017 to December, 2019 (described below in 'Sample collection').

Sample collection. In the first cohort study, a 9-ml venous blood sample was collected from each participant. Maternal serum samples were obtained at baseline (time of admission), at $2 \mathrm{~h}$ postpartum and on postpartum day 3. In the second cohort study, placental tissue and amniotic fluid samples were obtained during cesarean deliveries. Urine samples $(n=7)$ of neonates without complications were obtained within 2 days after birth at term. Serum and urine samples were separated by centrifugation at $1,400 \mathrm{x}$ g for $5 \mathrm{~min}$ at room temperature and stored at $-80^{\circ} \mathrm{C}$ until tested. A pairwise comparison of SCCA measurements between maternal serum and neonatal urine could not be performed as they were obtained from separate cases. Amniotic fluid samples were aseptically retrieved by amniotomy following uterine muscle layer incision. These samples were divided into 2 groups as follows: Control samples with clear amniotic fluid $(n=27)$ and samples of meconium-stained amniotic fluid $(n=4)$. Formalin-fixed, paraffin-embedded tissue sections from 3 full-term placentas and 3 cervical cancer cases from the Department of Obstetrics and Gynecology of Nara Medical University Hospital were obtained from the pathology archives. A commercially available fetal skin tissue slide obtained from a 35-week fetus was purchased (Pantomics, Inc.). SCCA protein expression was assessed by immunohistochemistry.

Preparation of amniotic fluid samples. Amniotic fluid contains fetal skin keratinocytes, amnion epithelial cells, epithelioid cells, fibroblasts and pluripotent stem cells. Approximately $20 \mathrm{ml}$ amniotic fluid was obtained during surgery. As previously described (18), cell block sections of amniotic fluid samples were prepared using the sodium alginate method and subjected to hematoxylin and eosin (H\&E) and immunohistochemical staining. Cellular pellets were fixed with $10 \%$ paraformaldehyde for $12 \mathrm{~h}$ at room temperature. Formalin-fixed pellets were then embedded in paraffin. The unstained sections (3- $\mu \mathrm{m}$-thick) were stained with hematoxylin for $5 \mathrm{~min}$ and with eosin (Sakura Finetek Japan Co., Ltd.) for $2 \mathrm{~min}$ at room temperature. The slides were examined and photographed using an Olympus CX41 microscope (Olympus Corporation).

SCCA measurements. The serum SCCA levels were measured by competitive Luminex immunoassay using a commercially available kit from BML according to the manufacturer's instructions. SCCA levels were calculated using their average optical densities based on standard curves. The detection limit was $0.1 \mathrm{ng} / \mathrm{ml}$.

Immunohistochemistry. SCCA is a member of the ovalbumin serpin (ov-serpin)/clade B serpin family, which is composed of SCCA1 (SERPINB3) and SCCA2 (SERPINB4) $(19,20)$. Routine $\mathrm{H} \& \mathrm{E}$ staining followed by immunohistochemical staining was performed to detect SCCA protein expression. For immunohistochemistry, paraffin sections (3- $\mu \mathrm{m}$-thick) were mounted on poly-L-lysine-coated slides. Samples, including fetal skin, placental and cervical cancer tissues were incubated for $1 \mathrm{~h}$ at room temperature with a mouse-specific antibody against pan-cytokeratin AE1/AE3 (ab27988; Abcam; 1:100 dilution), 
Table I. Demographic characteristics of the patients in the present study.

\begin{tabular}{|c|c|c|c|c|}
\hline Characteristic & Normal vaginal delivery & CS with labor & CS without labor & P-value \\
\hline Number of deliveries & 339 & 28 & 97 & \\
\hline Maternal age (years) & & & & $<0.001$ \\
\hline Mean \pm SD & $32.1 \pm 5.6^{\mathrm{a}}$ & $34.8 \pm 4.9^{b}$ & $34.3 \pm 4.4^{\mathrm{c}}$ & \\
\hline Median (range) & $33.0(17-46)$ & $35.0(26-43)$ & $34.0(22-46)$ & \\
\hline Gestational age (weeks) & & & & $<0.001$ \\
\hline Mean \pm SD & $39.1 \pm 1.5^{\mathrm{d}}$ & $38.4 \pm 2.3^{\mathrm{e}}$ & $37.6 \pm 1.5^{\mathrm{f}}$ & \\
\hline Median (range) & $39(26-41)$ & $38.0(29-41)$ & $38.0(29-41)$ & \\
\hline Parity & & & & $<0.001$ \\
\hline 0 & 174 & 20 & 24 & \\
\hline$\geq 1$ & 165 & 8 & 73 & \\
\hline
\end{tabular}

All P-values were obtained using the Kruskal-Wallis test. a vs. b, $\mathrm{P}=0.054$; b vs. c, $\mathrm{P}=0.999$; a vs. c, $\mathrm{P}=0.002$; $\mathrm{d}$ vs. e, $\mathrm{P}=0.134$; e vs. f, $\mathrm{P}=0.001$; and $\mathrm{d}$ vs. $\mathrm{f}, \mathrm{P}<0.001$, determined by the Kruskal-Wallis test with post hoc analysis with Bonferroni correction. CS, cesarean section.

Table II. SCCA levels for each delivery mode at the time of admission, at $2 \mathrm{~h}$ postpartum, and on postpartum day 3.

\begin{tabular}{lccc}
\hline Delivery mode & Admission & 2 h postpartum & Postpartum day 3 \\
\hline Vaginal (n) & 231 & 277 & 150 \\
Mean \pm SD & $1.83 \pm 1.86^{\mathrm{a}}$ & $4.15 \pm 2.42^{\mathrm{b}}$ & $0.96 \pm 0.99^{\mathrm{c}}$ \\
Median (range) & $1.60(0.40-19.30)$ & $3.70(0.60-18.80)$ & $0.8(0.40-11.80)$ \\
CS with labor (n) & 23 & 14 & 8 \\
Mean \pm SD & $1.78 \pm 1.35^{\mathrm{d}}$ & $1.83 \pm 1.05^{\mathrm{e}}$ & $0.78 \pm 0.16^{\mathrm{f}}$ \\
Median (range) & $1.40(0.70-6.80)$ & $1.60(0.80-4.20)$ & $0.85(0.50-0.90)$ \\
CS without labor (n) & 70 & 60 & 53 \\
Mean \pm SD & $1.58 \pm 1.82^{\mathrm{g}}$ & $1.43 \pm 0.56^{\mathrm{h}}$ & $0.85 \pm 0.53^{\mathrm{i}}$ \\
Median (range) & $1.30(0.60-16.10)$ & $1.30(0.60-3.40)$ & $0.80(0.40-3.70)$
\end{tabular}

All $\mathrm{P}$-values were obtained using the Kruskal-Wallis test. a vs. b, $\mathrm{P}<0.001$; b vs. c, $\mathrm{P}<0.001$; a vs. c, $\mathrm{P}<0.001$; $\mathrm{d}$ vs. e, $\mathrm{P}=0.999$; e vs. f, $\mathrm{P}=0.003$; d vs. f, $\mathrm{P}=0.002$; g vs. h, $\mathrm{P}=0.999$; h vs. i, $\mathrm{P}<0.001$; and g vs. i, $\mathrm{P}<0.001$, determined by the Kruskal-Wallis test with post hoc analysis with Bonferroni correction. CS, cesarean section; SCCA, squamous cell carcinoma antigen.

monoclonal antibodies against SCCA1/A2 (sc-28384; Santa Cruz Biotechnology, Inc.; 1:100 dilution), or normal rabbit IgG (\#3900; Cell Signaling Technology, Inc.; 1:100 dilution). The sections were placed for $30 \mathrm{~min}$ at room temperature with amplification reagent (Simple Stain MAX PO; Nichirei Biosciences), followed by incubation for $5 \mathrm{~min}$ with diaminobenzidine (Simple Stain DAB Solution, Nichirei Bioscience).

Statistical analysis. Statistical analyses were performed using SPSS Statistics version 25 (IBM Japan). Data distribution was verified by the Shapiro-Wilk test, indicating that all groups exhibited non-normal distribution (data not shown). Data were analyzed using the Kruskal-Wallis test followed by post hoc analysis with Bonferroni correction to evaluate differences among the 3 groups in maternal age, gestational age of the fetus and SCCA level between baseline, $2 \mathrm{~h}$ postpartum and 3 day postpartum. Pearson's Chi-squared test was performed to compare parity among the 3 groups. P-values of $<0.05$ were considered to indicate statistically significant differences.

\section{Results}

The patient demographic characteristics are presented in Table I. There were significant differences between the vaginal delivery and the 2 cesarean section groups as regards maternal age, gestational age and parity (all $\mathrm{P}<0.001)$. Maternal age was lower, whereas gestational age was higher in the vaginal delivery group than in the cesarean section groups. In the cesarean section with labor group, $71.4 \%$ of mothers (20/28) were nulliparous compared with $51.3 \%$ (174/339) in the vaginal delivery group and $24.7 \%$ (24/97) in the cesarean section without labor group. The numbers of serum samples at baseline, at $2 \mathrm{~h}$ postpartum and on postpartum day 3 are presented in Table II. The serum SCCA levels in all groups were significantly altered during the study period. In the vaginal delivery group, the SCCA levels significantly increased from baseline to $2 \mathrm{~h}$ postpartum, and decreased 3 day postpartum. The SCCA levels were lowest at 3 days postpartum. In both cesarean section groups, the serum SCCA levels at baseline and $2 \mathrm{~h}$ postpartum did not differ 
Table III. SCCA levels in amniotic fluid and neonatal urine.

SCCA levels (mean \pm SD)

Clear amniotic fluid $(n=27)$

Meconium-stained amniotic fluid $(\mathrm{n}=4)$

Neonatal urine $(n=7)$

P-value

$569.37 \pm 432.32$

$378.08 \pm 57.82$

$322.83 \pm 427.74$

0.147

P-value was obtained using the Kruskal-Wallis test.

A

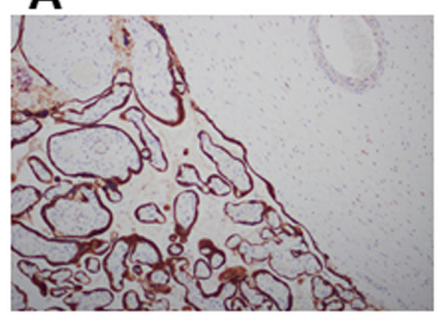

D

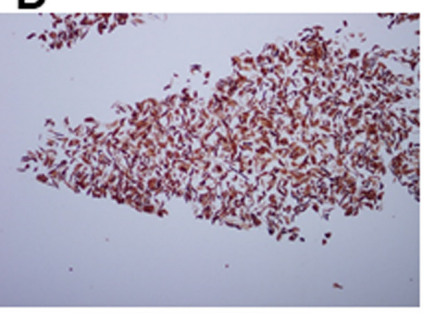

G
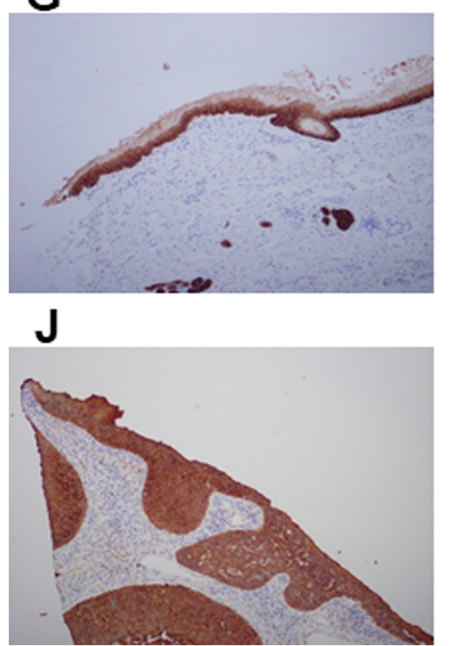

B

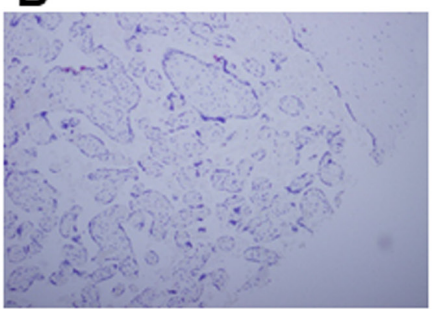

E

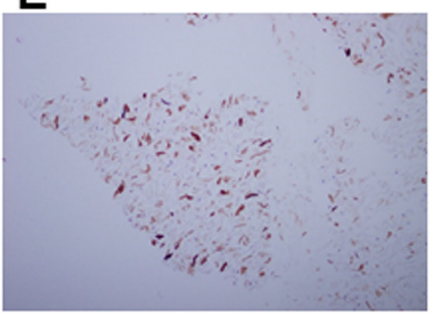

H

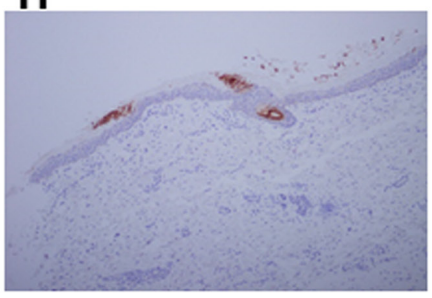

K

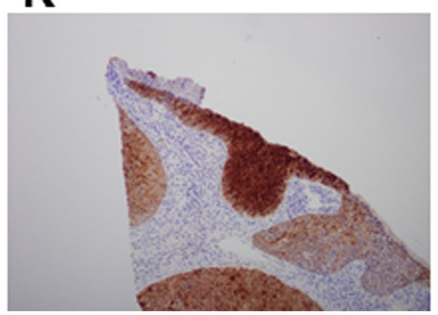

C

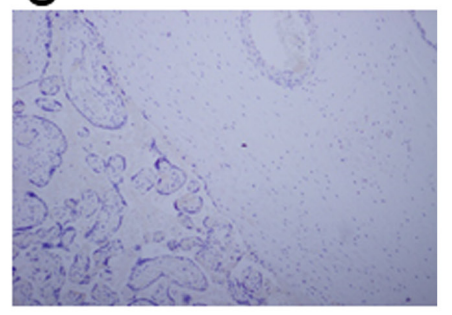

$\mathbf{F}$

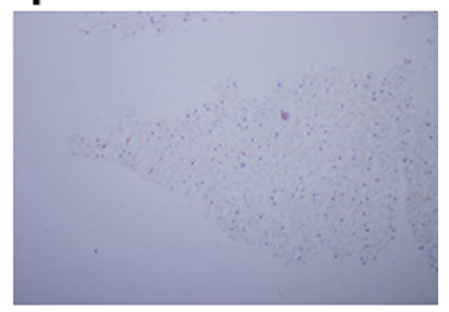

I

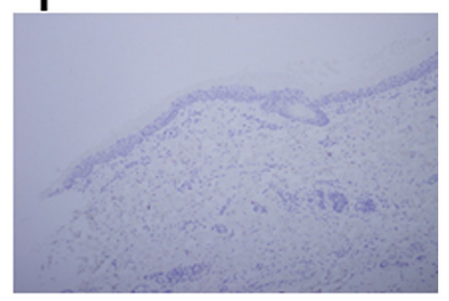

$\mathbf{L}$

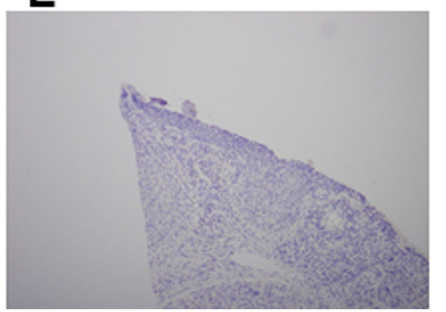

Figure 1. Distribution of cytokeratin and squamous cell carcinoma antigen (SCCA) in full-term placentas, amniotic fluid cell blocks, fetal skin, and cervical cancer tissues. The distribution of (A, D, G and J) cytokeratin, (B, E, H and K) SCCA, and (C, F, I and L) normal rabbit IgG in the (A, B and C) full-term placentas, (D, E and F) amniotic fluid cell blocks, $(\mathrm{G}, \mathrm{H}$ and $\mathrm{I})$ fetal skin and $(\mathrm{J}, \mathrm{K}$ and $\mathrm{L})$ cervical cancer tissues is shown by representative immunostaining. The full-term placenta was (A) positive for cytokeratin AE1/AE3 and (B) negative for SCCA (magnification, x100). Cytokeratins were present in the epidermis and all kinds of trophoblasts. Amniotic fluid cell blocks exhibited (D) intense labeling for AE1/AE3, but (E) weak and scattered staining for SCCA (magnification, x100). Fetal skin epidermal cells were negatively labeled for SCCA. SCCA was localized in scattered cells of the keratinization area and hair matrix cells. As positive controls for immunostaining, cervical cancer tissues were positively labeled for (J) cytokeratin and (K) SCCA (magnification, $\mathrm{x} 100$ ).

significantly $(\mathrm{P}=0.999$ and 0.999 , respectively). All groups exhibited a marked decrease in SCCA levels from admission or $2 \mathrm{~h}$ postpartum to 3 days postpartum $(\mathrm{P}<0.001$ or $\mathrm{P}<0.001$ for the vaginal delivery group, $\mathrm{P}=0.002$ or $\mathrm{P}=0.003$ for the cesarean section with labor group, and $\mathrm{P}<0.001$ or $\mathrm{P}<0.001$ for the cesarean section without labor group; Table II).

The origin of SCCA in amniotic fluid was then determined. No significant differences were observed in the SCCA levels between the clear amniotic fluid, meconium-stained amniotic fluid and neonatal urine samples, all of which were extremely high (Table III). Finally, the present study investigated whether SCCA was expressed in the placenta, amniotic fluid cells and fetal skin. Full-term placentas, amniotic fluid cell blocks, fetal skin and cervical cancer tissues were examined by immunostaining for cytokeratin and SCCA (Fig. 1). Cytokeratin is widely used as a marker for the identification 
and characterization of epithelial cells, trophoblasts and cervical cancer cells (Fig. 1A, D, G and J). For SCCA immunostaining, cervical cancer tissues were used as the positive control (Fig. 1K). The samples were reviewed by experienced pathologists (Tomoko Uchiyama and Chiho Ohbayashi, Department of Diagnostic Pathology, Nara Medical University, Kashihara, Japan) to determine the expression of cytokeratin, SCCA and nonimmune IgG. The amniotic membrane, and trophoblast cells and mesenchymal cells in the placenta were not stained for SCCA (Fig. 1B). Diffuse cytokeratin immunostaining was strong in the majority of amniotic fluid cells (Fig. 1D), whereas SCCA expression was scattered and weakly positive (Fig. 1E). Fetal skin epidermal cells were positively labeled for cytokeratin (Fig. 1G), but SCCA expression was limited (Fig. 1H). In fetal skin, SCCA was localized to scattered cells of the keratinization area (stratum corneum) and hair matrix cells (Fig. 1H).

\section{Discussion}

The present study suggests, for the first time, to the best of our knowledge, that amniotic fluid components containing SCCA enter the maternal circulation during normal vaginal delivery, and that amniotic fluid SCCA may originate from fetal urine.

First, SCCA levels in amniotic fluid were reportedly much higher than those in maternal serum, suggesting the potential clinical utility of serum SCCA level as a marker for AFE diagnosis $(10,13)$. This fact suggests that amniotic fluid components enter the maternal circulation in patients with AFE. However, it remains unclear whether normal delivery affects the influx of amniotic fluid components into the maternal circulation. The present study therefore determined the dynamic changes in maternal serum SCCA levels before and after delivery in relation to the mode of delivery. In vaginal delivery, serum SCCA levels increased significantly from baseline to $2 \mathrm{~h}$ postpartum and decreased 3 days postpartum (Table II). The decline in serum SCCA levels 3 days after delivery may be due to metabolism of SCCA in the kidney and its short half-life (2). The disappearance curve of serum SCCA levels following resection of malignant tumor has exhibited an average half-life of $2.2 \mathrm{~h}$ (21). It was found that amniotic fluid components entered the maternal circulation in all cases of vaginal delivery, but no women exhibited cardiorespiratory symptoms suggestive of AFE.

Second, it was hypothesized that during a cesarean section, maternal serum SCCA levels should increase as the influx of amniotic fluid into the maternal circulation is inevitable. Unexpectedly, maternal serum SCCA levels remained unaltered at $2 \mathrm{~h}$ postpartum compared with those at baseline in both cesarean patients with or without labor. However, the SCCA levels in patients with normal delivery were significantly higher than those of planned cesarean patients without labor $(1.83 \pm 1.86 \mathrm{ng} / \mathrm{ml}$ vs. $1.58 \pm 1.82 \mathrm{ng} / \mathrm{ml}, \mathrm{P}=0.033$; Table II). Furthermore, the SCCA levels were extremely high in those who reached full dilatation and delivered vaginally $(4.15 \pm 2.42 \mathrm{ng} / \mathrm{ml})$. These data suggest that the onset of labor is a major factor for elevated SCCA levels, and that amniotic fluid can enter the maternal circulation from a micro-laceration at the amniotic fluid-maternal blood barrier when the cervix is fully opened. Benson et al suggested (16) that amniotic fluid, fetal cells, hair and other debris can enter the maternal circulation by breaching the placenta-uterine vascular circulation barrier during full cervical dilatation. That is, both the onset of labor and full cervical dilatation are the causes of entry of amniotic fluid components into the maternal circulation.

Third, the present study investigated the origin of amniotic fluid SCCA. There was no significant difference in SCCA levels between amniotic fluid with and without meconium staining (Table III), suggesting that meconium is not a source of amniotic fluid SCCA. The placenta was negative for SCCA immunostaining. Okawa et al reported that the cytoplasm of adult skin epidermal cells was positively stained for SCCA (22), whereas SCCA was not expressed in the granular, superficial spinous, deep spinous and basal layers of fetal skin. This difference may be due to insufficient differentiation of fetal skin epidermal cells. With careful observation, SCCA-positive cells can be focally detected in the keratinized layer of fetal skin. Furthermore, the immunostaining of amniotic fluid cells revealed scattered SCCA positivity, which may indicate that SCCA-positive keratinocytes are released into the amniotic fluid. These results suggest that amniotic fluid SCCA is unlikely to be derived from placenta, amniotic fluid cells and meconium. SCCA levels in amniotic fluid may originate from fetal urine, as neonatal urine SCCA levels were nearly as high as those in amniotic fluid samples (Table III). However, the exact origin of SCCA in fetal urine remains unknown. Thus, studies on the origin of SCCA suggest that keratinized fetal skin, placenta and meconium are not primary sources, whereas the fetal urine is more likely. However, it does not deny the possibility other than the above.

Fourth, serum biomarkers including ZnCP-1, STN, IGFBP1 and SCCA are not used widely in clinical practice due to several limitations including low sensitivity, lack of standardization of methods, difficulty of measuring markers during the acute phase of an episode, and the fact that its use remains experimental (4-13). The diagnostic significance of SCCA in AFE has been well-documented recently (13). The authors previously reported that serum SCCA levels were 25-fold higher in women with autopsy-proven AFE (mean, $112.0 \mathrm{ng} / \mathrm{ml}$ ) than in healthy controls with normal deliveries (mean, $4.4 \mathrm{ng} / \mathrm{ml}$ ) (13). The SCCA test can improve discriminatory power in predicting AFE (sensitivity, 60.0\%; specificity, 89.2\%) (13). The disastrous amniotic fluid entry into the maternal circulation may lead to dramatic sequelae of clinical events including cardiopulmonary collapse and coagulopathy in autopsy-proven AFE. The association between amniotic fluid entry into the maternal circulation and a rapid onset of anaphylaxis may explain the underlying mechanism of the pathogenesis of AFE. In the present study, however, despite the reliable amniotic fluid entry into the maternal circulation during normal vaginal delivery, no case of concomitant anaphylactic reaction was found. However, the reasons why they did not develop a fatal anaphylactic shock are not clear. There are two possibilities for this: AFE does not develop unless a certain amount of amniotic fluid enters the maternal circulation and an idiosyncrasy may be related to AFE onset. Amniotic fluid SCCA may become a useful marker for identifying amniotic fluid entry into the maternal circulation rather than supporting the potential diagnosis of AFE (23). Notwithstanding these limitations, serum SCCA 
levels are abnormally elevated among women with established AFE (13). Currently, serodiagnosis of AFE based on amniotic fluid-specific markers is not internationally accepted.

Finally, there are two limitations to the present study. The first is that as this was a single-center study, it may have been subjected to unpredictable selection bias. In addition, our hospital is a university hospital; therefore, there may have been bias regarding more serious disease cases being present. Secondly, the number of emergency cesarean section cases was relatively small, which needs to be investigated in future large-scale studies.

In conclusion, the present study demonstrates that dynamic changes in maternal serum SCCA levels during labor indicate that amniotic fluid components can enter the maternal circulation during normal vaginal delivery. The onset of labor and full cervical dilatation are the main causes of entry of amniotic fluid components into the maternal circulation. The origin of SCCA may be fetal urine, but it does not rule out other possibilities. Amniotic fluid SCCA, which may be derived from fetal urine, can enter the maternal circulation during vaginal delivery. The onset of labor and full cervical dilatation are the main causes of entry of amniotic fluid components into the maternal circulation.

\section{Acknowledgements}

The authors acknowledge the outstanding histological assistance of Dr Takeshi Nishikawa, Dr Sumire Sugimoto and Dr Tomoko Uchiyama (Department of Diagnostic Pathology, Nara Medical University, Kashihara, Japan).

\section{Funding}

The present study was supported by a Grant-in-Aid for Scientific Research from the Ministry of Education, Science, and Culture of Japan to the Department of Obstetrics and Gynecology, Nara Medical University (JSPS KAKENHI grant no. JP16K11150; JSPS KAKENHI grant no. JP 15K10682; JSPS KAKENHI grant no. JP 17K11292, and JSPS KAKENHI grant no. JP 26462497). In addition, the present study was supported by AMED under grant no. 17gk0210005h0003 and by the Tohoku Bureau of Economy, Trade and Industry (grant no. Tohoku 1607028) and the Kanzawa Medical Research Foundation (grant no. 30-28).

\section{Availability of data and materials}

All data generated or analyzed during this study are included in this published article or are available from the corresponding author on reasonable request.

\section{Authors' contributions}

$\mathrm{HK}, \mathrm{NK}$ and KN contributed to the conception and design of the study. KN, NK and YY collected patient data and serum and tissue samples. KN, NK and YY measured the ELISA samples. The samples were reviewed by experienced pathologists (LL and CO) to determine the expression of cytokeratin and SCCA. KN, NK and HK were involved in the drafting of the manuscript or revising it critically for important intellectual content. The final version of the manuscript has been read and approved by all authors.

\section{Ethics approval and consent to participate}

All procedures involving human participants were in accordance with the ethical standards of the institutional research committee and with the 1964 Helsinki Declaration and its later amendments or comparable ethical standards. The protocols were approved by the ethics committee of Nara Medical University (approval numbers 579, 579-2, 873, and 873-2). The present study is a retrospective observational study, carried out by the opt-out method of our hospital website. Written informed consent was obtained from each study subject, and all subjects consented to donate samples.

\section{Patient consent for publication}

Not applicable.

\section{Competing interests}

The authors declare that they have no competing interests.

\section{References}

1. Conde-Agudelo A and Romero R: Amniotic fluid embolism: An evidence-based review. Am J Obstet Gynecol 201: 445.e1-445. e13, 2009.

2. Kobayashi H: Amniotic fluid embolism: Anaphylactic reactions with idiosyncratic adverse response. Obstet Gynecol Surv 70: 511-517, 2015.

3. Schneider SS, Schick C, Fish KE, Miller E, Pena JC, Treter SD, Hui SM and Silverman GA: A serine proteinase inhibitor locus at $18 q 21.3$ contains a tandem duplication of the human squamous cell carcinoma antigen gene. Proc Natl Acad Sci USA 92: 3147-3151, 1995.

4. Kanayama N, Yamazaki T, Naruse H, Sumimoto K, Horiuchi K and Terao T: Determining zinc coproporphyrin in maternal plasma - a new method for diagnosing amniotic fluid embolism. Clin Chem 38: 526-529, 1992.

5. Kobayashi H, Ohi H and Terao T: A simple, noninvasive, sensitive method for diagnosis of amniotic fluid embolism by monoclonal antibody TKH-2 that recognizes NeuAc alpha 2-6GalNAc. Am J Obstet Gynecol 168: 848-853, 1993.

6. Kobayashi H, Ooi H, Hayakawa H, Arai T, Matsuda Y, Gotoh K and Tarao T: Histological diagnosis of amniotic fluid embolism by monoclonal antibody TKH-2 that recognizes NeuAc alpha 2-6GalNAc epitope. Hum Pathol 28: 428-433, 1997.

7. Oi H, Kobayashi H, Hirashima Y, Yamazaki T, Kobayashi T and Terao T: Serological and immunohistochemical diagnosis of amniotic fluid embolism. Semin Thromb Hemost 24: 479-484, 1998.

8. Oi H, Naruse K, Noguchi T, Sado T, Kimura S, Kanayama N, Terao T and Kobayashi H: Fatal factors of clinical manifestations and laboratory testing in patients with amniotic fluid embolism. Gynecol Obstet Invest 70: 138-144, 2010.

9. Tsunemi T, Oi H, Sado T, Naruse K, Noguch T and Kobayashi H: An overview of amniotic fluid embolism: Past, present and future directions. Open Womens Health J 6: 24-29, 2012.

10. Naruse K, Noguchi T, Yoshida S, Tsunemi T, Shigetomi H, $\mathrm{Oi} \mathrm{H}$ and Kobayashi H: Identification of interleukin-6 (IL-6) and squamous cell carcinoma (SCC) as amniotic fluid-specific markers. Open J Obstet Gynecol 2: 147-150, 2012.

11. Iwai K, Oi H, Tsunemi T, Naruse K, Noguchi T, Sado T and Kobayashi H: Sialyl Tn and Zinc coproporphyrin 1 as potential serum markers of amniotic fluid embolism. Adv Obstet Gynecol 63: 483-487, 2011 (In Japanese).

12. Legrand M, Rossignol M, Dreux S, Luton D, Ventré C, Barranger E, Laribi S, Payen D and Muller F: Diagnostic accuracy of insulin-like growth factor binding protein-1 for amniotic fluid embolism. Crit Care Med 40: 2059-2063, 2012. 
13. Koike N, Oi H, Naruse K, Kanayama N and Kobayashi $\mathrm{H}$ : Squamous cell carcinoma antigen as a novel candidate marker for amniotic fluid embolism. J Obstet Gynaecol Res 43: 1815-1820, 2017.

14. Kato H: Expression and function of squamous cell carcinoma antigen. Anticancer Res 16: 2149-2153, 1996.

15. Ullman E, Pan JA and Zong WX: Squamous cell carcinoma antigen 1 promotes caspase-8-mediated apoptosis in response to endoplasmic reticulum stress while inhibiting necrosis induced by lysosomal injury. Mol Cell Biol 31: 2902-2919, 2011.

16. Benson MD, Cheema N, Kaufman MW, Goldschmidt RA and Beaumont JL: Uterine intravascular fetal material and coagulopathy at peripartum hysterectomy. Gynecol Obstet Invest 73 158-161, 2012.

17. Karakas B, Qubbaj W, Al-Hassan S and Coskun S: Noninvasive digital detection of fetal DNA in plasma of 4-week-pregnant women following in vitro fertilization and embryo transfer. PLoS One 10: e0126501, 2015.

18. Kase S, Namba K, Iwata D, Mizuuchi K, Kitaichi N, Tagawa Y, Okada-Kanno H, Matsuno Y and Ishida S: Diagnostic efficacy of cell block method for vitreoretinal lymphoma. Diagn Pathol 11: 29, 2016.

19. Cataltepe S, Gornstein ER, Schick C, Kamachi Y, Chatson K, Fries J, Silverman GA and Upton MP: Co-expression of the squamous cell carcinoma antigens 1 and 2 in normal adult human tissues and squamous cell carcinomas. J Histochem Cytochem 48: $113-122,2000$.
20. Izuhara K, Yamaguchi Y, Ohta S, Nunomura S, Nanri Y, Azuma Y, Nomura N, Noguchi Y and Aihara M: Squamous cell carcinoma antigen 2 (SCCA2, SERPINB4): An emerging biomarker for skin inflammatory diseases. Int J Mol Sci 19: 1102, 2018.

21. Yoshimasu T, Maebeya S, Suzuma T, Bessho T, Tanino H, Arimoto J, Sakurai T and Naito Y: Disappearance curves for tumor markers after resection of intrathoracic malignancies. Int J Biol Markers 14: 99-105, 1999.

22. Okawa T, Yamaguchi Y, Kou K, Ono J, Azuma Y, Komitsu N, Inoue Y, Kohno M, Matsukura S, Kambara T, et al: Serum levels of squamous cell carcinoma antigens 1 and 2 reflect disease severity and clinical type of atopic dermatitis in adult patients. Allergol Int 67: 124-130, 2018.

23. Kobayashi $\mathrm{H}$ : The entry of fetal and amniotic fluid components into the uterine vessel circulation leads to sterile inflammatory processes during parturition. Front Immunol 3: 321, 2012.

This work is licensed under a Creative Commons Attribution-NonCommercial-NoDerivatives 4.0 International (CC BY-NC-ND 4.0) License. 